\title{
A Study on the Influence of Mobile Phone Addiction on Academic Burnout in Postgraduate Students with Mediating Effect of Sleep Quality
}

\author{
Yuzi Cheng ${ }^{1^{*}}$, Zonghe Zhang ${ }^{2, a}$ \\ ${ }^{1,2}$ Student work department, Nanfang College of Sun Yat-Sen University, Guangzhou, Guangdong, 510970, China
}

\begin{abstract}
In order to understand the current situation of postgraduates' sleep quality, mobile phone addiction and learning burnout, and at the same time explore the mechanism of the influence of mobile phone addiction and sleep quality on academic burnout. The study used the "Mobile Phone Addiction Index Scale", "Pittsburgh Sleep Quality Index Scale", and "Graduate Academic Burnout Scale". A total of 673 graduate students were surveyed. There are significant differences in mobile phone addiction among graduate students in different genders, majors, grades and family economic conditions. The sleep quality of postgraduate students will be different due to different grade and family economic conditions. The academic burnout level of postgraduate students will be different due to different genders, majors, grades, family economics, and whether they have work experience. The sleep quality of graduate students plays a partly mediating effect between mobile phone addiction and academic burnout.
\end{abstract}

\section{INTRODUCTION}

Mobile phone users excessively use mobile phones for some reasons, and then appear psychological and physical symptoms of discomfort, which is defined as mobile phone addiction1. Domestic research on mobile phone dependence of college students shows that the phenomenon of college students' mobile phone dependence is very common[1][2][3][4]. Sleep quality is a comprehensive evaluation of sleep processes and effects. It is based on objective sleep conditions, plus subjective feelings as the main subject of comprehensive evaluation results. Scholars have made a lot of research results on sleep quality, but the research groups are mostly special populations, such as students, the elderly, soldiers, and medical staff. Related research results show that the sleep quality of these people is worse than ordinary people[5][6]. Academic burnout is the negative psychology and behavior of students who are tired of learning due to longterm academic pressure, and lack motivation and interest in learning[7] .

Studies have shown that sleep quality is affected by mobile phone addiction[8][9][10]. Some studies have shown that there is a certain relationship between students' academic performance and sleep quality[11][12][13]. Other studies have shown that mobile phone addiction will directly affect students' learning burnout[14][15]. So what role does sleep quality play in the impact of mobile phone addiction on academic burnout? To explore the role of sleep quality in the influence of mobile phone addiction on academic burnout, which is helpful for in-depth understanding of the mechanism of mobile phone addiction on academic burnout. At the same time, it can also help prevent and reduce the adverse effects of academic burnout and protect students' body and mind. Provide basis and guidance for health.

\section{STUDYDESIGN}

In this study, a total of 700 questionnaires were distributed to graduate students. After the recovery, 27 invalid questionnaires were eliminated, resulting in a total of 673 questionnaires.

The research tools are as follows:

The Pittsburgh Sleep Quality Index (PSQI) is used to measure sleep quality. The higher the score is, the worse the sleep quality is. The internal consistency coefficient of the scale is 0.84 , the split-half reliability is 0.87 , and the test-retest reliability is 0.81 .

The Mobile Phone Addiction Index (MPAI) is used to measure the level of mobile phone addiction. The scale was compiled by Professor Liang Yongchi of the University of Hong Kong based on the diagnostic criteria for addiction in DSM-4. The Cronbach coefficient is 0.87 .

The academic burnout questionnaire for graduate students is used to measure the level of academic burnout. The internal consistency coefficient of the questionnaire is above 0.7 , and the split-half reliability is above 0.6 .

\footnotetext{
*Corresponding Author. Email: 316459641@qq.com

azonghe.zhang@outlook.com
} 


\section{RESEARCHRESULT}

\subsection{Influencing factors of mobile phone addiction among graduate students}

The results of the study show that there are significant differences in mobile phone addiction among graduate students in different genders, majors, grades, and family economics. Among them, women have higher scores on mobile phone addiction than men, and students in literature and history have higher scores on mobile phone addiction than students in science and engineering. High, Yan1's mobile phone addiction is significantly higher than Yan2, and Yan2 is significantly higher than Yan3. The better the family economy is, the lower the mobile phone addiction is. (Yan1= first-year graduate school student; Yan2 $=$ second-year graduate school student; Yan3= thirdyear graduate school student)

Table1. Analysis of the Influence of Mobile Phone Addiction in postgraduate students

\begin{tabular}{lll}
\hline & $\boldsymbol{t} / \boldsymbol{F}$ & $\boldsymbol{p}$ \\
\hline genders & $-6.46^{* *}$ & 0.00 \\
majors & $6.9^{* *}$ & 0.00 \\
grades & $16.78^{* *}$ & 0.00 \\
family & $4.34^{* *}$ & 0.00 \\
economics & & \\
\hline $\mathrm{p}<0.01, * \mathrm{p}<0.05$ & &
\end{tabular}

\subsection{Influencing factors of graduate students' sleep quality}

The results show that the total sleep quality scores of graduate students have significant differences in grades and family economics. The sleep quality of Yan3 students is better than that of Yan1 and Yan2 students. The better the family economic situation is, the better the sleep quality of students is.

Table2. Analysis of the Influence of Sleep Quality in postgraduate students

\begin{tabular}{lll}
\hline & $\boldsymbol{t} / \boldsymbol{F}$ & $\boldsymbol{p}$ \\
\hline grade & $11.93^{* *}$ & 0.00 \\
family economic & $4.52^{* *}$ & 0.00 \\
\hline $\mathrm{p}<0.01, * \mathrm{p}<0.05$ & &
\end{tabular}

\subsection{Influencing factors of postgraduate academic burnout}

The results show that the total score of postgraduate academic burnout is significantly different in gender, work experience, major, grade, and family economic situation. There is no significant difference in birthplace and whether it is an only child. Among them, women are higher than men and have no jobs. Those without experience are higher than those with work experience, and those with history are higher than those with science and engineering. The level of academic burnout of Yan1 students in graduate school is lower than that of Yan1 and Yan2 students. The better the family financial situation is, the lower the level of academic burnout is.

Table3. Analysis of the Influence of Academic Burnout in postgraduate students

\begin{tabular}{|c|c|c|}
\hline & $t / F$ & $p$ \\
\hline gender & $-3.46^{* *}$ & 0.00 \\
\hline major & $3.137 * *$ & 0.00 \\
\hline $\begin{array}{l}\text { work } \\
\text { experience }\end{array}$ & $-2.94 * *$ & 0.00 \\
\hline grade & $14.23 * *$ & 0.00 \\
\hline $\begin{array}{l}\text { family } \\
\text { economic }\end{array}$ & $4.73 * *$ & 0.00 \\
\hline
\end{tabular}

\subsection{The mediating role of sleep quality in the influence of mobile phone addiction on academic burnout}

\subsubsection{Correlation analysis of postgraduates' mobile phone addiction, sleep quality and academic burnout}

The results show that there is a significant positive correlation between graduate students' sleep quality, mobile phone addiction and academic burnout.

Table4. Analysis of the correlation between the study variables

\begin{tabular}{lcll}
\hline & 1 & 2 & 3 \\
\hline 1.sleep quality & & \\
2.mobile phone addiction & $0.24^{* *}$ & \\
3.academic burnout & $0.3^{* *}$ & $0.51^{* *}$ & \\
\hline$* \mathrm{p}<0.01, * \mathrm{p}<0.05$ & &
\end{tabular}

3.4.2 The mediating role of sleep quality in the influence of mobile phone addiction on academic burnout

This study used sequential testing regression coefficients to test the mediating effect. The results showed that mobile phone addiction in graduate students significantly positively affects academic burnout and sleep quality. As the level of mobile phone addiction increases, the level of academic burnout will increase and sleep quality will deteriorate. After adding the intermediary variable sleep quality, the regression of postgraduate academic burnout to mobile phone addiction is still significant, and sleep quality plays a part of the mediating role in the relationship between mobile phone addiction and academic burnout. That is, mobile phone addiction not only directly affects 
academic burnout, but also affects the level of academic burnout by affecting the quality of sleep.

Table5. Mediating Effect of Sleep Quality on Mobile Phone Addiction and Academic Burnout

\begin{tabular}{ccccccc}
\hline & $\begin{array}{c}\text { Standardized } \\
\text { regression equation }\end{array}$ & $\mathbf{R}^{2}$ & $\mathbf{B}$ & $\mathbf{S E}$ & Beta & $t$ \\
\hline 1 & $\mathrm{Y}=0.514 \mathrm{X}$ & 0.264 & 0.422 & 1.253 & 0.514 & $15.541^{* * *}$ \\
2 & $\mathrm{M}=0.238 \mathrm{X}$ & 0.055 & 0.059 & 0.009 & 0.238 & $6.345^{* * *}$ \\
& $\mathrm{Y}=0.297 \mathrm{M}$ & 0.087 & 0.983 & 0.122 & 0.297 & $8.071^{* * *}$ \\
\multirow{2}{*}{3} & $\mathrm{Y}=0.186 \mathrm{M}$ & 0.295 & 0.386 & 0.027 & 0.47 & $14.104^{* * *}$ \\
& $+0.47 \mathrm{X}$ & & 0.613 & 0.11 & 0.186 & $5.565^{* *}$ \\
\hline$* * \mathrm{p}<0.001,{ }^{* *} \mathrm{p}<0.01,{ }^{*} \mathrm{p}<0.05$ & & & & &
\end{tabular}

\section{DISCUSSION}

This research reveals the status quo of mobile phone addiction, sleep quality and learning burnout among graduate students. There are significant differences in the level of mobile phone addiction among graduate students of different genders, majors, grades and family economic status. There are significant differences in sleep quality among graduate students of different grades and family economic conditions. There are significant differences in the level of academic burnout of graduate students in different genders, majors, grades, family economics, and whether they have work experience.

Mobile phone addiction and sleep quality are both important factors affecting academic burnout, and sleep quality mediates the effect of mobile phone addiction on academic burnout. When a graduate student's mobile phone addiction is higher, his sleep quality will be worse, and poor sleep quality will lead to further student burnout. So if you want to reduce the academic burnout of graduate students, on the one hand, you can achieve this goal by directly reducing the mobile phone addiction of graduate students, and on the other hand, you can achieve this goal by improving the quality of sleep of graduate students. For example, schools should strengthen postgraduate education on the use of mobile phones to make postgraduates aware of the dangers of mobile phone addiction. At the same time, lectures on related sleep hygiene education will be conducted to let students understand which factors are harmful to sleep and which factors promote sleep, so that students can consciously improve sleep quality.

\section{REFERENCES}

1. Qu Mingyu. Investigation and countermeasures on mobile phone dependence of college students. [J]. Journal of Chengdu Aeronautical Vocational and Technical College, 2016,3:11-13.

2. Wang Chang, Wang Shengyong, Li Wenhao, et al. Investigation on the mobile phone dependence syndrome of 2213 college students in Guangzhou.
[J].Chinese Journal of Epidemiology, 2013,34(10): 949-952.

3. Billieux J. Problematic Use of the Mobile Phone: A literature review and a pathways model[J]. Current Psychiatry Reviews,2012, 8:299-307.

4. Qu Xiaoting, Cao Mingrui, Li Xiaobo, et al. Sleep in the elderly and its influencing factors[J]. Chinese Journal of Gerontology,2011,3(31): 852-853.

5. Chen Baifeng, Ying Xia, Fang Lu, Li Fan, Yue Na. The relationship between smartphone addiction and sleep and depression among college students in a medical college $[\mathrm{J}]$. Journal of Chifeng University,2016,32(3):191-193

6. Sun Ning. Research on College Students' Academic Burnout and Its Countermeasures[J]. Journal of Hotan Demonstration College,2010, 29(64):70-71.

7. Sahin S, Ozdemir K, Unsal A \& et al.Evaluation of mobile phone addiction level and sleep quality in university students $[\mathrm{J}]$. Park J Med Sci,2014,29(4),913-918.

8. Zhu Yuxin. The relationship between mobile phone addiction and sleep quality in college students[J]. Social Psychological Science, 2015,10:73-80.

9. Wang Haiqing, Rao Jiaming, Ye Yunfeng, et al. Association analysis of mobile phone use and sleep quality among college students in a university in Guangzhou[J]. Practical preventive medicine , 2016,23(4):429-433.

10. Zhang Shangxiao, Yang Xiaoyan, Zhang Yong, et al. Analysis of the correlation between sleep quality and learning burnout of college students in a medical college in Xinjiang[J]. Chinese Occupational Medicine, 2016,43(2):181-184.

11. Ni Jing, Chen Weijuan, Luo Guangyu, et al. Discuss the impact of medical freshmen's sleep quality on academic burnout and coping strategies[J]. Health Vocational Education,2016,18:118-120.

12. Yogesh S, Abha S \&Pryanka. Mobile usage and patterns among medical students[J]. Indian J Physiol Pharmacol,2014,58(1):100-103.

13. Arbabisarjou A, Hashemi SM, Sharif MR \& et al. The 
Relationship between Sleep Quality and Social Intimacy, and Academic Burn-Out in Students of Medical Sciences[J]. Glob J Heslth Sci,2016, 8(5):116-118.

14. Liu Tingting. Research on the relationship between mobile phone addiction and sleep and academic work of medical college students. Chinese Journal of Drug Dependence,2017,26(1):33-37.

15. Ge Xuhua. The relationship between mobile phone addiction and learning burnout in secondary vocational school students. Mental health education in primary and secondary schools,2013,15:14-15. 